\title{
Étude de l'additivité de la dégradation in sacco de la matière sèche des mélanges simples d'aliments concentrés
}

\author{
P Chapoutot, S Giger, D Sauvant, S Jeantet \\ Station de Nutrition et Alimentation (INRA) de I'INA-PG, \\ 16, rue C-Bernard, 75231 Paris Cedex 05, France
}

\begin{abstract}
Summary - Barley and maize were combined with either lupine seeds or corn gluten meal so that the 4 blends present different rumen degradation rates for their carbohydrate and/or nitrogen constituents. The measured values (dm) of dry matter in sacco degradability of these concentrates were higher before and lower after $8 \mathrm{~h}$ of incubation compared to the calculated values of degradability (dc) obtained by the additive method according to their composition. Moreover, the differences (dm dc) were the highest for the less degradable feeds.
\end{abstract}

Introduction - Les résultats de dégradation in sacco des constituants des matières premières sont appliqués aux aliments composés en supposant que les phénomènes de dégradation sont additifs. La présente étude a pour objectif de vérifier cette hypothèse dans le cas de mélanges simples d'aliments concentrés.

Matériel et Méthodes - Quatre matières premières ont été choisies a priori pour leur richesse en fractions rapidement ou lentement dégradables dans le rumen : orge $(O)$ vs maïs (M) pour les glucides, lupin (L) vs corn gluten meal (G) pour l'azote. Celles-ci ont été combinées 2 à 2 au sein de mélanges de façon à synchroniser ou dissocier dans le temps la disponibilité des glucides et de l'azote pour les microorganismes du rumen $(\mathrm{OL}=40 \% \mathrm{O}+$ $50 \% \mathrm{~L} ; \mathrm{ML}=40 \% \mathrm{M}+50 \% \mathrm{~L} ; \mathrm{OG}=60 \% \mathrm{O}+$ $30 \%$ G; MG $=60 \% M+30 \% \mathrm{G} ;+6 \%$ mélasse $+4 \%$ complément minéral et vitaminisé (CMV) dans chacun). Les 4 matières premières et les 4 mélanges simples ont fait l'objet de mesures de dégradation de la MS dans le rumen de vaches fistulées selon la méthodologie décrite par Michalet-Doreau et al (1987).

Résultats et Discussion - Les valeurs moyennes de dégradation de la MS $(\mathrm{dm})$ sont présentées au tableau I. A chaque temps, à partir des données mesurées sur les 4 matières premières, des valeurs calculées de dégradation de la MS des mélanges (dc) ont été obtenues par additivité en tenant compte de leur composition centésimale et d'une dégradabilité totale de la mélasse et du CMV. Dans le court terme (avant $8 \mathrm{~h}$ d'incubation), les $d m$ sont significativement plus élevées que les $d c$, les écarts absolus ( $d m-d c$ ) étant d'autant plus importants que la dégradabilité de la MS est plus faible. Par contre, dans le moyen et le long terme, les dégradabilités observées sont légèrement plus faibles que celles obtenues par le calcul additif. L'impact sur la dégradabilité théorique de la MS (DTms), calculée selon la méthode de Kristensen et al (1982) en tenant compte d'un taux de renouvellement des particules du rumen de $6 \% / \mathrm{h}$ et d'un même taux de disparition de la MS du sachet entre 0 et 2 h qu'entre 2 et $4 \mathrm{~h}$, est relativement limité pour les aliments $\mathrm{OL}$, $\mathrm{ML}$ et $\mathrm{OG}$ car les 2 phénomènes décrits ci-dessus ont tendance à se compenser. Par contre, dans le cas de l'aliment MG contenant les matières premières les 
Tableau I. Dégradabilité de la MS des aliments concentrés.

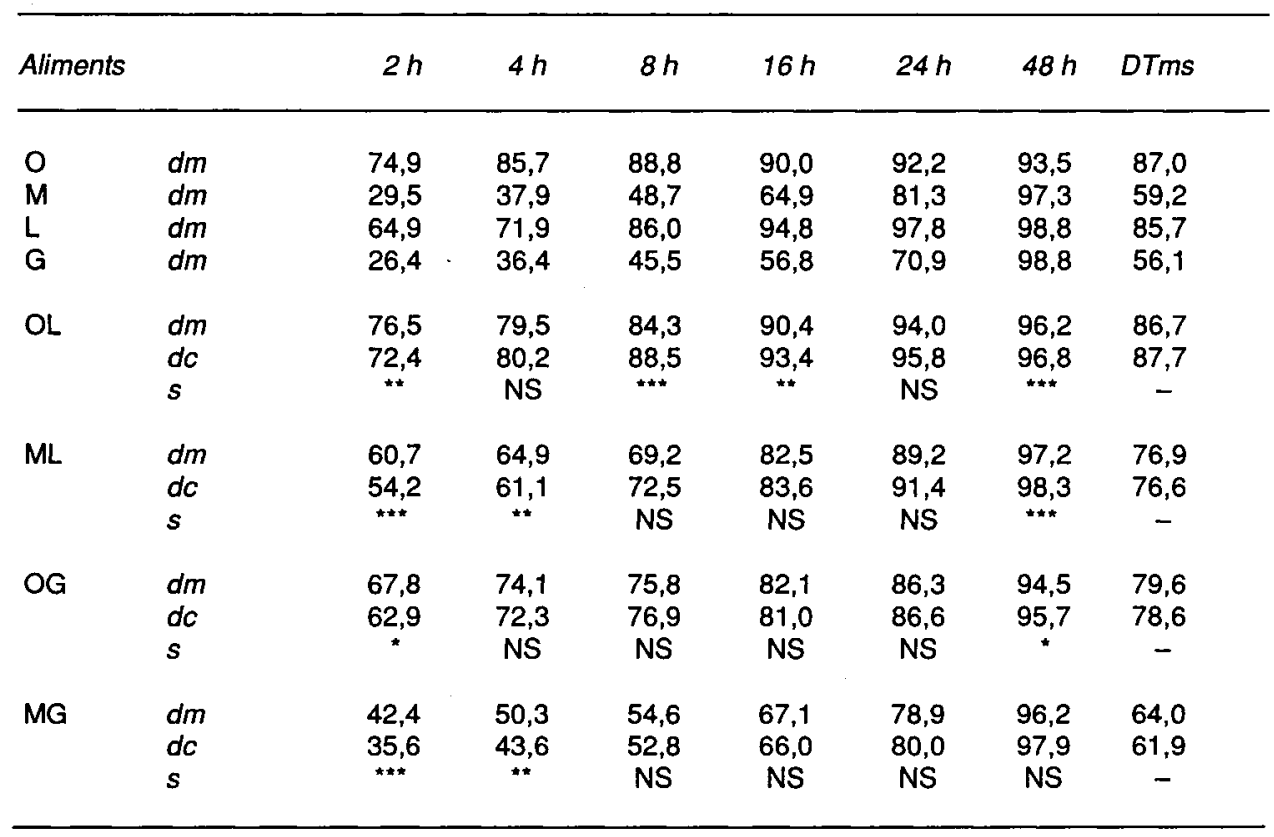

$d m$ : dégradabilité mesurée; $d c$ : dégradabilité calculée; $s$ : niveau de signification $\left({ }^{* * *}=0,001,{ }^{* *}=0,01,{ }^{*}=0,05\right.$, NS = non significatif).

moins dégradables, la DTms issue des valeurs mesurées demeure supérieure de 2 points à celle obtenue à partir des valeurs calculées par additivité.

II apparaît donc que les mélanges puissent favoriser l'activité microbienne du contenu des sachets par un meilleur équilibre des substrats. Ce phénomène sem- blerait être d'autant plus sensible qu'on associe des ingrédients peu solubles et digestibles à court terme.

Michalet-Doreau $B$, Vérité $R$, Chapoutot $\mathrm{P}$ (1987) Bull Tech CRVZ Theix INRA 69, 5-7

Kristensen ES, Moller PD, Hvelplund T (1982) Acta Agric Scand 32, 123-127 